\title{
The 4D Camera: Very High Speed Electron Counting for 4D-STEM
}

Jim Ciston $^{1 *}$, Ian J. Johnson ${ }^{2}$, Brent R. Draney ${ }^{3}$, Peter Ercius ${ }^{1}$, Erin Fong ${ }^{2}$, Azriel Goldschmidt ${ }^{2}$, John M. Joseph $^{2}$, Jason R. Lee ${ }^{3}$, Alexander Mueller ${ }^{1}$, Colin Ophus ${ }^{1}$, Ashwin Selvarajan ${ }^{3}$, David E. Skinner ${ }^{3}$, Thorsten Stezelberger ${ }^{2}$, Craig S. Tindall ${ }^{2}$ Andrew M. Minor ${ }^{1,4}$ and Peter Denes ${ }^{5}$

1. National Center for Electron Microscopy, Molecular Foundry, Lawrence Berkeley National

Laboratory, Berkeley, California, USA.

2. Engineering Division, Lawrence Berkeley National Laboratory, Berkeley, California, USA.

3. National Energy Research Scientific Computing Center, Lawrence Berkeley National Laboratory Berkeley, California, USA.

4. Department of Materials Science and Engineering, University of California, Berkeley, California, USA.

5. Lawrence Berkeley National Laboratory, Berkeley, California, USA.

* Corresponding author: JCiston@lbl.gov

A vast array of new experimental modalities have been enabled in the past several years through the development of pixelated detectors synchronized to probe scanning electronics. Such camera systems can then acquire the rich information present in the central portion of the convergent beam electron diffraction pattern as a function of probe position (4D-STEM). These 4-dimensional (or more) datasets can be readily exploited for phase contrast ptychographic imaging [1], nanoscale strain mapping [2], unit cell resolution quantitative scanning position averaged convergent beam electron diffraction [3], and more. While such detectors are now commercially available from several manufacturers with single electron sensitivity, they are typically limited to approximately 1 millisecond $(1 \mathrm{kHz})$ readout times [4] while conventional integrating-detector HAADF STEM image data is acquired at approximately 10 microsecond $(100 \mathrm{kHz})$ scan rates. This speed constraint places significant limits on accessible fields of view at high resolution due to sample drift, and limits in-situ acquisition to a $4 \mathrm{D}$ frame rate of $\sim 1$ minute.

We present here the development, installation, and characterization of the 4D Camera, a CMOS Active Pixel Sensor that consists of a $576 \times 576$ array of $10 \mu \mathrm{m}$ pixels [5] of a design related to the original TEAM detector [6] and an outer HAADF detector with 16 concentric quadrant diodes (Figure 1). Full-frame data from this sensor is read out at $87 \mathrm{kHz}$, digitized locally at the camera head, and sent over 96 multi-gigabit optical links to 4 Field Programmable Gate Array (FPGA) modules for image assembly, packetization, and routing. In initial tests, the sensor exhibited single electron sensitivity from at accelerating voltages from $<30 \mathrm{keV}$ to $300 \mathrm{keV}$, enabling electron counting methods to effectively eliminate detector readout noise. Initial data has been acquired using a structured mask cut by focused ion beam from a $50 \mathrm{~nm} \mathrm{SiN}$ film coated with $1000 \mathrm{~nm}$ of evaporated gold (Figure 2).

All data will be streamed in real time via a $400 \mathrm{Gbps} 1 \mathrm{~km}$ optical link to the Cori supercomputer at the National Energy Research Scientific Computing Center (NERSC), which will perform the 4-dimensional reconstruction and HDF5 file writing before additional asynchronous processing and analysis. By design this is a parallel computational workflow, and NERSC's HPC provides concurrency and a rich software environment to scale up analysis and feedback codes. In-hardware edge-computing on these FPGA devices may also be used to carry out initial data processing (e.g. gain and dark correction, thresholding) before the data is placed on the network. 
Compression factors of more than 100 are expected when analog signals from the detector are converted to electron events, often referred to as electron counting. Machine learning routines based on convolutional neural networks are under development for more reliable and efficient detection and clusterization of electron strikes on the detector. Live reconstruction of integrating detectors with user-defined shape (e.g. annular bright field, split quadrant detectors, etc.) as a function of scan position may also be calculated at the detector in firmware and provide instantaneous feedback to the user on a lower bandwidth (10 gigabit) display channel. More resource intensive data processing and reduction algorithms and phase contrast imaging methods such as matched illumination and detector interferometry [7] and ptychography will be performed at NERSC with the goal of near-live asynchronous feedback to the user [8].

\section{References:}

[1] PD Nellist, BC McCallum and JM Rodenburg, Nature 374 (1995), p. 630.

[2] VB Ozdol et al., Applied Physics Letters 106 (2015), p. 253107.

[3] C Ophus et al., Applied Physics Letters 110 (2017), p. 063102.

[4] MW Tate et al., Microscopy \& Microanalysis 22 (2016), p. 237.

[5] IJ Johnson et al., Microscopy \& Microanalysis 24 (S1) (2018), p. 166.

[6] M Battaglia et al., Nucl. Inst. and Methods in Phys. Res. A 622(3) (2010), p. 669.

[7] C Ophus et al., Nature Communications 7 (2016), p. 10719.

[8] This work was supported by the Office of Science, Office of Basic Energy Sciences, of the U.S. Department of Energy under Contract No. DE-AC02-05CH11231. Research was performed at the Molecular Foundry and the National Energy Research Scientific Computing Center, DOE Office of Science User Facilities. JC and CO acknowledge support from the DOE Early Career Research Program.

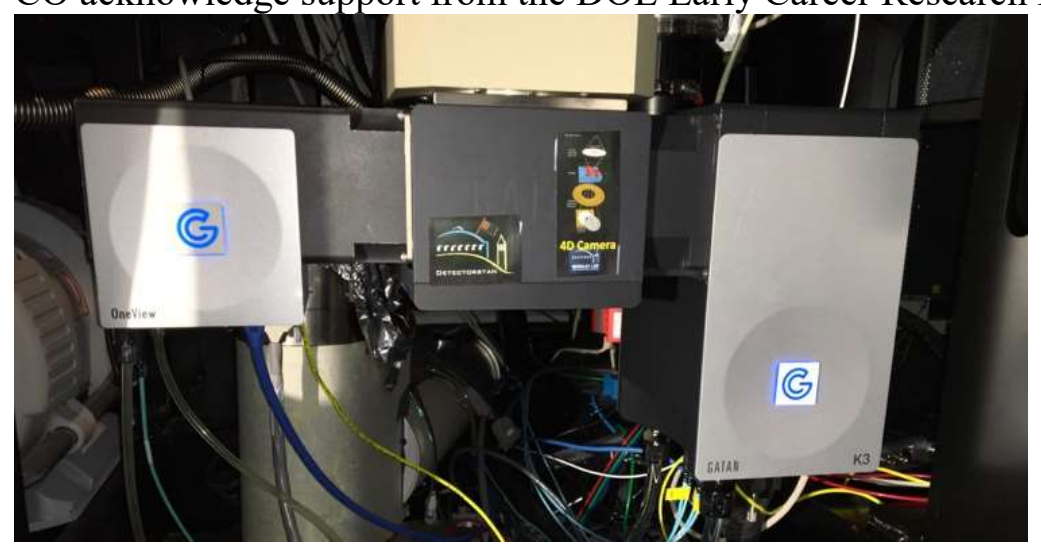

Figure 1. Optical photograph of installed detector system

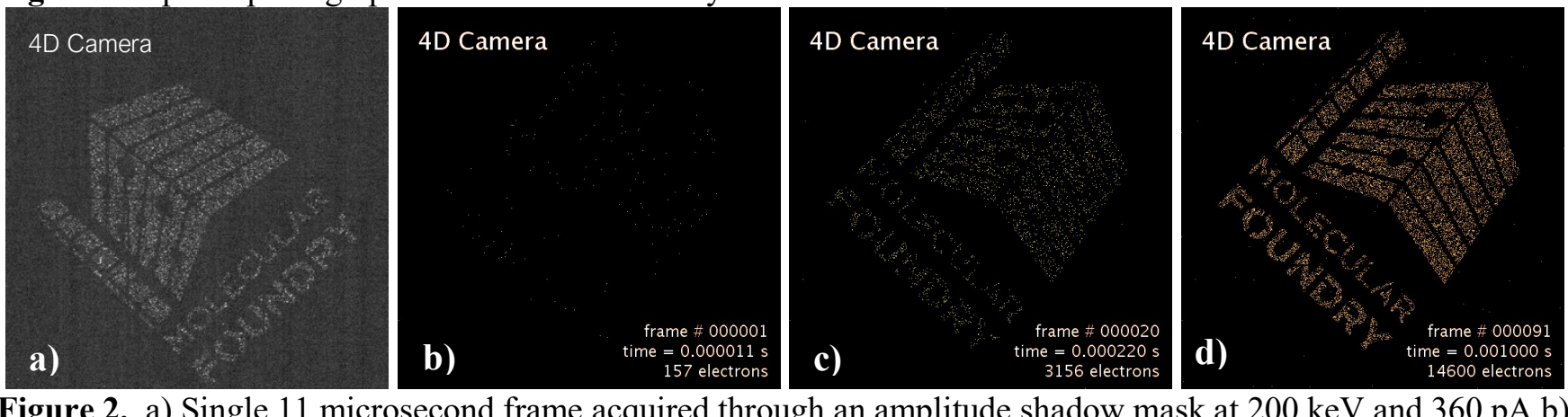

Figure 2. a) Single 11 microsecond frame acquired through an amplitude shadow mask at $200 \mathrm{keV}$ and $360 \mathrm{pA} \mathrm{b}$ ) Single 11 microsecond frame acquired through an amplitude shadow mask at $200 \mathrm{keV}$ and $20 \mathrm{pA}$ with electron strikes detected at a threshold criteria of 6-sigma above the dark noise c) Sum of 20 electron strike-thresholded frames $(0.22 \mathrm{~ms}$ total sensor illumination) at $200 \mathrm{keV}$ and $20 \mathrm{pA} \mathrm{d})$ Sum of 91 electron strike-thresholded frames (1.00 ms total sensor illumination) at $200 \mathrm{keV}$ and $20 \mathrm{pA}$ 